\title{
La nefropatia in corso di malattia di Fabry
}

\author{
Renzo Mignani \\ UO Nefrologia e Dialisi, Ospedale Infermi, Rimini
}

\begin{abstract}
Diagnosis and management of nephropathy in Fabry disease
Nephropathy is a life-threatening aspect of Fabry disease. The signs and symptoms start in childhood and adolescence with albuminuria and proteinuria. If not treated, patients with renal involvement progress to chronic renal failure and rapidly to end-stage renal disease necessitating dialysis or kidney transplant. The early start of enzyme replacement therapy has proved effective in reducing GL3 deposits in renal cells and reversing albuminuria and proteinuria, thereby stabilizing renal function. Instead, delayed introduction of agalsidase therapy fails to decrease proteinuria and to arrest the progression of renal failure towards end-stage renal disease. We propose guidelines and recommendations for the diagnosis and management of Fabry nephropathy in adults based on the consensus of opinion of the participants in the International Fabry Nephropathy Roundtable of 2007 and the KDIGO consensus conference of 2015.
\end{abstract}

Keywords: Chronic renal failure, End-stage renal disease, Fabry disease, Nephropathy

\section{Il danno renale nella malattia di Fabry}

Il coinvolgimento renale nella forma classica della malattia di Fabry esordisce in genere nell'adolescenza con albuminuria e proteinuria. Tali alterazioni sono l'espressione del deposito di substrato nei vari elementi cellulari del nefrone, dalle cellule endoteliali, ai podociti, alle cellule mesangiali e tubulari. Recenti studi istologici hanno però dimostrato come in realtà il deposito di globotriaosylceramide (GL3) nei podociti sia presente già in utero e nei bambini prima ancora della comparsa di albuminuria e progredisca con l'età. Da qui l'importanza di eseguire lo studio bioptico del rene, il solo in grado di definire l'estensione e l'entità del danno renale. Al contrario, nei pazienti con varianti atipiche, per lo più con un prevalente danno cardiaco, i reperti urinari e l'insufficienza renale possono essere anche del tutto assenti sino alla quarta/quinta decade (1).

La proteinuria nel paziente con malattia di Fabry è in genere modesta ma aumenta con l'insorgenza e il progredire

Accepted: February 12, 2017

Published online: July 28, 2017

Indirizzo per la corrispondenza:

Dr. Renzo Mignani

UO Nefrologia e Dialisi

Ospedale Infermi

Viale Luigi Settembrini, 2

47923 Rimini

renzo.mignani@ausIromagna.it dell'insufficienza renale senza raggiungere però l'entità della proteinuria nefrosica. L'ipertensione arteriosa non è un sintomo tipico della malattia, anche se ampi studi di registro hanno osservato una prevalenza di ipertensione nel $25 \%$ dei pazienti arruolati (2). La terapia enzimatica sostitutiva (TES) ha dimostrato sia in studi randomizzati che nelle analisi dei registri una scarsa capacità di ridurre la proteinuria in tali pazienti. Al contrario, se all'agalsidasi si associa un farmaco attivo sul sistema renina-aldosterone è possibile ridurre in modo significativo la proteinuria e rallentare altrettanto la progressione della malattia. Con l'impiego di ACE inibitori o antagonisti dell'angiotensina, associati o no alla terapia enzimatica, oltre a ottenersi la riduzione della proteinuria a livelli fisiologici, è possibile rallentare significativamente la progressione dell'insufficienza renale soprattutto se l'avvio della terapia antiproteinurica e della TES sono precoci, iniziati già con valori di proteinuria di 300-400 mg/die (3).

\section{Il paziente con insufficienza renale cronica}

Nel paziente che non inizia precocemente la terapia enzimatica la progressione del danno renale è molto rapida. Dopo la comparsa di proteinuria, già nella seconda/terza decade di vita si manifesta una insufficienza renale cronica (IRC) che a sua volta progredisce rapidamente verso la fase terminale e l'esaurimento funzionale dei reni. Tale progressione è sovrapponibile a quella riscontrata nella nefropatia diabetica ed è altrettanto rapida nei maschi come nelle femmine. La comparsa dell'IRC determina un progressivo aumento della proteinuria e una sempre più frequente ipertensione arteriosa (4). Pertanto, è 
fondamentale in tali pazienti il trattamento della proteinuria con farmaci antiproteinurici. In questa fase il paziente in genere continua a lamentare i segni e i sintomi extrarenali della malattia, in particolare quelli espressione del danno neurologico e cardiologico che tendono anch'essi a progredire.

\section{II paziente in terapia renale sostitutiva}

Il paziente che raggiunge la fase terminale dell'IRC necessita dell'introduzione di una terapia sostitutiva renale. Nell'immediato, il trattamento dialitico, peritoneale o extracorporeo rappresenta il rimedio salvavita come in tutti i pazienti uremici. Nei pazienti con malattia di Fabry che iniziano la dialisi la sintomatologia extrarenale (come i dolori) di solito persiste e progredisce come accade per la cardiomiopatia perché all'accumulo di substrato nei cardiomiociti si associa il sovraccarico idrico che il dializzato manifesta a causa all'anuria. Alcuni studi osservazionali o basati sui dati di registro hanno infatti dimostrato nei pazienti in dialisi una elevata comorbidità soprattutto cardiaca e cerebrovascolare responsabile di una sopravvivenza media inferiore a quella dei pazienti diabetici e non diabetici in dialisi $(5,6)$. Al contrario, nei pazienti con trapianto renale i dolori regrediscono spontaneamente dopo poche settimane dall'intervento, probabilmente per effetto dell'attività enzimatica presente nel rene ricevuto e non affetto (6). Inoltre in tali pazienti anche la cardiomiopatia progredisce più lentamente: ecco perché nei pazienti che raggiungono la fase terminale dell'IRC andrebbe incoraggiato, quando possibile, il trapianto renale pre-emptive evitando cosi la elevata comorbidità del paziente con malattia di Fabry in trattamento dialitico.

\section{Gli effetti della terapia enzimatica sulla nefropatia}

La terapia enzimatica sostitutiva (TES) con agalsidasi alfa o beta ha dimostrato la capacità di ridurre la progressione della nefropatia, soprattutto se instaurata precocemente. L'inizio della terapia in bambini e adolescenti con albuminuria e proteinuria ha consentito non solo la normalizzazione dei reperti urinari ma anche la pressoché completa rimozione dei depositi intracellulari di substrato $\operatorname{GL} 3(7,8)$. Al contrario, se I'avvio della terapia enzimatica avviene tardivamente quando è già presente un lieve deterioramento della funzione renale (stadio 2-3 CKD), è possibile un iniziale rallentamento della progressione dell'IRC con uno slope del GFR inferiore a $-3 \mathrm{~mL} /$ min/anno (9). Ma se la TES viene avviata in pazienti con proteinuria $>1 \mathrm{~g} /$ die o con una sclerosi glomerulare $>50 \%$ alla biopsia renale la progressione del danno renale è pressoché sovrapponibile a quella dei pazienti che non sono trattati con agalsidasi (10).

Al momento non sono disponibili studi comparativi controllati e di lunga durata in cui sia stata confrontata la diversa efficacia delle due formulazioni di agalsidasi nel rallentare la progressione della nefropatia e dell'IRC. En- trambe le formulazioni sembrano in grado di prevenire o rallentare la comparsa e la progressione del danno renale quando avviate precocemente mentre non lo sono se iniziate in presenza di una proteinuria importante o di una IRC avanzata.

\section{Le raccomandazioni nefrologiche}

In questi ultimi anni sono comparse in letteratura diverse pubblicazioni riguardanti raccomandazioni nefrologiche sulla diagnosi, il follow-up e la terapia nei pazienti con malattia di Fabry (11-13). Tali raccomandazioni sono scaturite da una serie di consensus conferences alle quali hanno partecipato i principali esperti mondiali e nazionali sulla malattia. Dal punto di vista nefrologico tutte concordano in alcuni principali fondamenti. Anzitutto la diagnosi precoce è indispensabile per un avvio altrettanto precoce della terapia e uno screening familiare che faccia emergere ulteriori membri affetti dalla malattia. L'esame urine e la biopsia renale, con analisi del frustolo alla microscopia ottica ed elettronica, rappresentano le principali indagini diagnostiche per evidenziare e controllare nel tempo un eventuale danno renale. II monitoraggio del paziente con esame urine, creatininemia e volume di filtrato glomerulare (GFR) deve essere eseguito con cadenza semestrale sia nei pazienti affetti e in terapia enzimatica sia in quelli asintomatici e non ancora in trattamento. La terapia antiproteinurica va avviata precocemente in presenza di una proteinuria superiore a $300 \mathrm{mg} /$ die. La TES va avviata il più precocemente possibile per evitare la progressione del danno sistemico e renale in particolare.

\section{Disclosures}

Financial support: No financial support was received for this submission.

Conflict of interest: The author has no conflict of interest.

\section{Bibliografia}

1. Desnick RJ, loannou YA, Eng CM. $\alpha$-Galactosidase A deficiency: Fabry disease. In: Scriver CR, Sly WA, Beaudet AL, Valle D, eds. The metabolic and molecular bases of inherited disease. $8^{\text {th }}$ ed. New York: McGraw-Hill. 2001:3733-74.

2. Ortiz A, Oliveira JP, Waldek S, Warnock DG, Cianciaruso B, Wanner C. Fabry Registry. Nephropathy in males and females with Fabry disease: cross-sectional description of patients before treatment with enzyme replacement therapy. Nephrol Dial Transplant. 2008;23(5):1600-7.

3. Warnock DG, Thomas CP, Vujkovac B, et al. Antiproteinuric therapy and Fabry nephropathy: factors associated with preserved kidney function during agalsidase-beta therapy. J Med Genet. 2015;52(12):860-6.

4. Ortiz A, Cianciaruso B, Cizmarik $M$, et al. End-stage renal disease in patients with Fabry disease: natural history data from the Fabry Registry. Nephrol Dial Transplant. 2010;25(3): 769-75. 
5. Thadhani $\mathrm{R}$, Wolf $\mathrm{M}$, West $\mathrm{ML}$, et al. Patients with Fabry disease on dialysis in the United States. Kidney Int. 2002;61(1): 249-55.

6. Mignani R, Feriozzi S, Pisani A, et al. Agalsidase therapy in patients with Fabry disease on renal replacement therapy: $A$ nationwide study in Italy. Nephrol Dial Transplant. 2008;23(5): 1628-35.

7. Tøndel C, Bostad L, Hirth A, Svarstad E. Renal biopsy findings in children and adolescents with Fabry disease and minimal albuminuria. Am J Kidney Dis. 2008;51(5):767-76.

8. Tøndel C, Bostad L, Larsen KK, et al. Agalsidase benefits renal histology in young patients with Fabry disease. J Am Soc Nephrol. 2013;24(1):137-48.

9. Schiffmann R, Warnock DG, Banikazemi M, et al. Fabry disease: progression of nephropathy and prevalence of cardiac and cerebrovascular events before enzyme replacement therapy. Nephrol Dial Transpl. 2009;24(7):2102-11.
10. Germain D, Waldek S, Banikazemi M, et al. Sustained, longterm renal stabilization after 54 months of agalsidase-beta therapy in patients with Fabry disease. J Am Soc Nephrol. 2007;18(5):1547-57.

11. Ortiz A, Oliveira JP, Wanner C, Brenner BM, Waldek S, Warnock DG. Recommendations and guidelines for the diagnosis and treatment of Fabry nephropathy in adults. Nat Clin Pract Nephrol. 2008;4(6):327-36.

12. Mignani R, Gallieni M, Feriozzi S, Pisani A, Marziliano N, Morrone A. [The nephropathy in the Anderson-Fabry disease: new recommendations for the diagnosis, the follow-up and the therapy]. G Ital Nefrol. 2015;32(4):1-13. [Article in Italian].

13. Schiffmann R, Hughes DA, Linthorst GE, et al. for Conference Participants. Screening, diagnosis, and management of patients with Fabry disease: conclusions from a "Kidney Disease: Improving Global Outcomes" (KDIGO) Controversies Conference. Kidney Int. 2017;91(2):284-293. 